\title{
STUDIES ON RIEMANNIAN HOMOGENEOUS SPACES
}

\author{
KATSUMI NOMIZU
}

The purpose of the present paper is to give the details of the results announced in the C. R. notes [7], [8] and [9].

In I which corresponds to [7], we shall develop a method for the study of affine transformations of a Riemannian manifold and prove Lemma 5 which is fundamental for the results in I and II. This part of our work has been motivated by a result of $\mathrm{K}$. Yano [12] stating that the largest connected group of affine transformations of a compact orientable Riemannian manifold consists of isometries. Our method will have further applications to some other problems concerning or involving the connected group of isometries of a Riemannian manifold (for example, [10]).

In II which corresponds to [8], we deal with the decomposition and irreducibility of a Riemannian homogeneous space by the application of Lemma 5. We also prove a theorem concerning forms whose covariant derivatives are zero. In III which corresponds to [9], we study the holonomy algebra of an irreducible Riemannian homogeneous space. If $G / H$ is a homogeneous space of a connected Lie group with compact $H$ such that the linear isotropy group is irreducible, then $G / H$ admits a unique invariant Riemannian connection. The problem to determine its holonomy algebra in terms of the Lie algebras of $G$ and $H$ was raised by Chevalley as well as by Lichnerowicz. We shall solve this problem by using the method of our previous work [6].

All the theorems in II and III can be considered as generalisations of the well known results of $\mathrm{E}$. Cartan on symmetric Riemannian homogeneous spaces. In this sense, the present paper is a continuation of [6] in which we have studied some other generalizations of Cartan's theory.

I would like to express my sincere thanks to Professor Lichnerowicz for his kind interest in my work and for many valuable suggestions with which he has encouraged me.

Received March 3, 1955. 


\section{Affine transformations of a Riemannian manifold}

I. Lemmas on affine transformations. In the following, let $M$ be a Riemannian manifold, or more precisely, a connected differentiable ${ }^{1 /}$ manifold with a positive definite Riemannian metric defined on it. The Riemannian metric on $M$ gives rise to an inner product $(X, Y)_{p}$ on the tangent space $T_{p}$ at each point $p$ of $M$. We speak of $T_{p}$ as a euclidean space with respect to this inner product. To the given Riemannian metric on $M$, there is associated a unique affine connection whose torsion tensor is zero and whose parallel displacement is isometric, that is, the parallel displacement along any (piece-wise differentiable) curve between two points $p$ and $q$ is an isometric linear mapping of the tangent space $T_{p}$ onto $T_{q}$. This affine connection is called the Riemannian connection, or Levi-Civita connection, associated to the given Riemannian metric.

By an affine transformation of $M$, we shall mean a differentiable transformation $\varphi$ of $M$ onto itself which leaves invariant the Riemannian connection [6]. It may be defined in the following intuitive fashion. Let $\tau$ be any curve from $p$ to $q$ and let $\varphi(\tau)$ be the curve which is the image of $\tau$ by $\varphi$. Denoting the parallel displacement along a curve by the same letter as the curve, $\varphi$ is called affine at $p$ if

$$
\varphi \cdot \tau \cdot X=\varphi(\tau) \cdot \varphi \cdot X, \text { for every } X \in T_{p},
$$

where $\varphi$ denotes the differential ${ }^{2)}$ of the transformation $\varphi . \quad \varphi$ is an affine transformation of $M$ if it is affine at every point of $M$.

A differentiable transformation $\varphi$ of $M$ is called isometric at a point $p$ if the differential of $\varphi$ is an isometric mapping of $T_{p}$ onto $T_{\varphi(p)}$, that is, $(\varphi X, \varphi Y)_{p(p)}$ $=(X, Y)_{p}$ for any $X$ and $Y \in T_{p} . \quad \varphi$ is called an isometry of $M$ if it is isometric at every point of $M$. Of course, this is equivalent to the usual definition.

LEMMA 1. Let $\varphi$ be an affine transformation of $M$. If $\varphi$ is isometric at a point $p$, then it is an isometry of $M$.

Proof. We show that $\varphi$ is isometric at any point $q$ of $M$. Let $\tau$ be any

1) By differentiability we understand that of class $\mathrm{C}^{\infty}$.

2) If $\phi$ is a differentiable mapping, the differential of $\phi$ at $p$ is the linear mapping of $T_{p}$ into $T_{\Phi_{(p)}}$ induced by $\phi$. In the following, we denote the differential of a mapping by the same letter, since there is no danger of confusion. 
curve from $q$ to $p$. Denoting the parallel displacement along $\tau$ by the same letter (which we shall do repeatedly in the sequel without further comment), we have

$$
\begin{aligned}
(X, Y)_{q} & =(\tau X, \tau Y)_{p}=(\varphi \cdot \tau X, \varphi \cdot \tau Y)_{p(p)} \\
& =(\varphi(\tau) \cdot \varphi X, \varphi(\tau) \cdot \varphi Y)_{\varphi(p)}=(\varphi X, \varphi Y)_{p(q)}
\end{aligned}
$$

for any $X, Y \in T_{q}$, which shows that $\varphi$ is isometric at $q$.

The homogeneous holonomy group $\Psi_{p}$ of $M$, with reference point at $p$, is a subgroup of the orthogonal group of $T_{p} . \quad M$ is called irreducible if $\Psi_{p}$ does not have any non-trivial invariant subspace; this notion is independent of the choice of $p$. Otherwise, it is called reducible and the orthogonal complement of an invariant subspace is also invariant by $\Psi_{p}$.

Lemma 2. Let $M$ be irreducible. If $\varphi$ is an affine transformation of $M$, then there exists a positive constant $c$ sush that $|\varphi X|=c|X|$ for every tangent vector $X$, where $|X|$ denotes the length of $X$, i.e., $X=(X, X)^{1 / 2}$.

Proof. Let $p$ be an arbitrary but fixed point of $M$. We introduce $(X, Y)^{*}$ $=(\varphi X, \varphi Y)_{\varphi(p)}$ for $X, Y \in T_{p}$, which is easily seen to be an inner product on $T_{p}$. Using the invariance of the inner product $(,)_{\varphi(p)}$ by the homogeneous holonomy group $\Psi_{p(p)}$, we have, for any element $\sigma$ of $\Psi_{p}$ represented by a closed curve $\sigma$ at $p$,

$$
\begin{aligned}
(\sigma X, \sigma Y)^{*} & =(\varphi \cdot \sigma X, \varphi \cdot \sigma Y)_{\varphi(p)}=(\varphi(\sigma) \cdot \varphi X, \varphi(\sigma) \cdot \varphi Y)_{\varphi(p)} \\
& =(\varphi X, \varphi Y)_{\varphi(p)}=(X, Y)^{*},
\end{aligned}
$$

which shows that the new inner product $(,)^{*}$ on $T_{p}$ is invariant by $\Psi_{p}$. $\Psi_{p}$ being irreducible, we see that there is a positive constant $c$ such that $(X, Y)^{*}$ $=c^{2}(X, X)_{p}$ for every $X, Y \in T_{p}$. Hence $|\varphi X|=c|X|$ for $X \in T_{p}$. For any other point $q$, let $\tau$ be an arbitrary curve from $q$ to $p$. If $Y \in T_{q}$ then $|\varphi \cdot Y|$ $=|\varphi(\tau) \cdot \varphi Y|=|\varphi \cdot \tau Y|=c|\tau Y|=c|Y|$.

If $M$ is reducible, we denote by $\Sigma$ the set of $\Psi_{p}$-invarant subspaces of $T_{p}$, where $p$ is an arbitrary but fixed point of $M$. We shall show that an affine transformation $\varphi$ of $M$ gives rise to a substitution of $\Sigma$ in the natural fashion. We take any arbitrary curve $\tau$ from $p$ to $\varphi(p)$ and consider the linear transformation $\rho=\tau^{-1} \cdot \varphi$ of $T_{p}$ onto itself (the succession of the differential of $\varphi$ and the parallel displacement $\tau^{-1}$ ). If $T^{\prime}$ is a subspace of $T_{p}$ invariant by $\Psi_{p}$, then 
the subspace $\rho \cdot T^{\prime}$ is invariant by $\Psi_{p}$. Indeed, if $\sigma$ is any element of $\Psi_{p}$ represented by a closed curve $\sigma$ at $p$, we have, for any $X \in T^{\prime}$,

$$
\begin{aligned}
\sigma \cdot \rho X & =\sigma \cdot \tau^{-1} \cdot \varphi \cdot X=\sigma \cdot \varphi \cdot \varphi^{-1}\left(\tau^{-1}\right) X \\
& =\varphi \cdot \varphi^{-1}(\sigma) \cdot \varphi^{-1}\left(\tau^{-1}\right) X \\
& =\varphi \cdot \varphi^{-1}\left(\tau^{-1}\right) \cdot \varphi^{-1}(\tau) \cdot \varphi^{-1}(\sigma) \cdot \varphi^{-1}\left(\tau^{-1}\right) X \\
& =\tau^{-1} \cdot \varphi \cdot \varphi^{-1}(\tau) \cdot \varphi^{-1}(\sigma) \cdot \varphi^{-1}\left(\tau^{-1}\right) X,
\end{aligned}
$$

where $\varphi^{-1}(\tau) \cdot \varphi^{-1}(\sigma) \cdot \varphi^{-1}\left(\tau^{-1}\right)$ is again an element of $\Psi_{p}$ represented by the corresponding closed curve at $p . T^{\prime}$ being invariant by $\Psi_{p}$, this element maps $X$ into some element $Y$ of $T^{4}$ and we have $\sigma \cdot \rho \cdot X=\rho \cdot Y \in \rho T^{\prime}$, which shows that $\rho T^{\prime}$ is invariant by $\Psi_{p}$.

The subspace $\rho T^{\prime}$ does not depend on the choice of a curve $\tau$ from $p$ to $\varphi(p)$ and is completely determined by $\varphi$. In fact, if $\tau^{\prime}$ is any other curve from $p$ to $\varphi(p)$, then we have

$$
\begin{aligned}
\tau^{-1} \cdot \varphi & =\tau^{\prime^{-1}} \cdot \tau^{\prime} \cdot \tau^{-1} \cdot \varphi=\tau^{\prime-1} \cdot \tau^{\prime} \cdot \varphi \cdot \varphi^{-1}\left(\tau^{-1}\right) \\
& =\tau^{\prime-1} \cdot \varphi \cdot \varphi^{-1}\left(\tau^{\prime}\right) \cdot \varphi^{-1}\left(\tau^{-1}\right),
\end{aligned}
$$

where $\varphi^{-1}\left(\tau^{\prime}\right) \cdot \varphi^{-1}\left(\tau^{-1}\right)=\varphi^{-1}\left(\tau^{\prime} \cdot \tau^{-1}\right)$ is an element of $\Psi_{p}$ and leaves $T^{\prime}$ invariant. This shows that $\tau^{-1} \cdot \varphi \cdot T^{\prime}=\tau^{\prime-1} \cdot \varphi \cdot T^{\prime}$.

We have thus seen that $\varphi$ gives rise to a substitution of $\Sigma$, which we denote by $s(\varphi)$. If $T^{\prime} \in \Sigma$, then $T^{\prime}$ and $s(\varphi) T^{\prime}$ are equivalent in the following sense: there is a linear transformation $\rho$ of $T^{\prime}$ onto $s(\varphi) T^{\prime}$ and an automorphism $\alpha$ of $\Psi_{p}$ such that $\rho(\sigma X)=\alpha(\sigma) \cdot \rho X$ for any $X \in T^{\prime}$ and $\sigma \in \Psi_{p}$. Indeed, we can take $\rho=\tau^{-1} \cdot \varphi$ for any curve from $p$ to $\varphi(p)$ and $\alpha(\sigma)=\varphi^{-1}\left(\tau \cdot \sigma \cdot \tau^{-1}\right)$ as we have already seen.

Now consider the group $A(M)$ of all affine transformations of $M$ and its identity component $A^{0}(M), A(M)$ is a Lie group [4].

LEMMA 3. The substitution $s(\varphi)$ of $\Sigma$ gives a representation of $A(M)$ : $s(\varphi \cdot \psi)=s(\varphi) \cdot s(\psi)$ for any $\varphi, \psi \in A(V)$.

Proof. Take a curve $\tau$ from $p$ to $\varphi(p)$ and a curve $\tau^{\prime}$ from $p$ to $\phi(p)$. Then $\varphi\left(\tau^{\prime}\right) \cdot \tau$ is a curve from $p$ to $\varphi \psi(p)$. We have

$$
\left(\varphi\left(\tau^{\prime}\right) \cdot \tau\right)^{-1} \cdot \varphi \cdot \psi=\tau^{-1} \cdot \varphi\left(\tau^{\prime-1}\right) \cdot \varphi \cdot \psi=\tau^{-1} \cdot \varphi \cdot \tau^{\prime-1} \cdot \psi \cdot
$$

Lemma 4. Let $\varphi_{t}$ be a one-parameter group of $A(V): \varphi_{t+s}=\varphi_{t} \cdot \varphi_{s}$. Then there is a one-paramter group $\rho_{t}$ of linear transformations of $T_{p}$ such that 
$\left(\rho_{t} X, Y\right)$ is continuous in $t$ and $X, Y \in T_{p}$ and such that $s\left(\varphi_{t}\right) \cdot T^{\prime}=\rho_{t} \cdot T^{\prime}$ for every $T^{\prime} \in \Sigma$.

Proof. For each $t$, we take a curve $\tau_{t}$ defined by $\tau_{t}(s)=\varphi_{s}(p), 0 \leqq s \leqq t$, and set $\rho_{t}=\tau_{t}^{-1} \cdot \varphi_{t}$.

Remark. In the case where $\varphi$ leaves $p$ invariant, we see that the substitution $s(\varphi)$ is induced by the linear isotropy induced by $\varphi$, that is, the linear transformation of $T_{p}$ induced by $\varphi$.

2. Canonical decomposition. If $T^{\prime}$ is a subspace of dimension $k$ invariant by $\Psi_{p}$, we get a distribution ( = field of subspaces) of dimension $k$ by the parallel displacement of $T^{\prime}$. This distribution is completely integrable. If $M^{\prime}$ is the maximal integral manifold through $p$ of this distribution, then $M^{\prime}$ has a Riemannian metric naturally induced by that of $M$.

G. de Rham has proved the following important results for a simply connected complete Riemannian manifold [11]:

A. If $T_{p}=T_{1}+T_{2}+\ldots+T_{r}$ is a decomposition of $T_{p}$ by $\Psi_{p}$ into mutually orthogonal invariant subspaces, then $M$ is the topological and Riemannian direct product $M_{1} \times M_{2} \times \ldots \times M_{r}$, where $M_{i}$ is the maximal integral manifold through $p$ of the distribution defined by $T_{i}$. $\Psi_{p}$ is the direct product $\Psi_{1} \times \Psi_{2}$ $\times \ldots \times \Psi_{r}$ where each $\Psi_{i}$ is isomorphic with the homogeneous holonomy group of $M_{i}$ and acts trivially on $T_{j}$ if $j \neq i$.

B. Let $T_{0}$ be the subspace of all elements $X \in T_{p}$ which are invariant by every element of $\Psi_{p}$. Let $T^{\prime}$ be the orthogonal complement of $T_{0}$ and decompose $T^{\prime}$ into the sum of mutually orthogonal irreducible invariant subspaces $T_{i}, i=1$, $2, \ldots, r$. In this way, we get a decomposition $T=T_{0}+T_{1}+\ldots+T_{r}$, where $T_{0}$ is the largest subspace of $T_{p}$ on which $\Psi_{p}$ acts trivially, each $T_{i}(i=1,2, \ldots$, $r)$ is irreducible and all $T_{i}(i=0,1, \ldots, r)$ are mutually orthogonal. This decomposition is unique. Or, more precisely, if $T^{\prime}$ is any invariant subspace, then either $T^{\prime}$ is orthogonal to $T_{i}$ or $T^{\prime}$ contains $T_{i}(i=1,2, \ldots, r)$.

We shall call the decomposition of $T_{p}$ (unique up to the order) indicated in $B$ the canonical decomposition of $T_{p}^{3}$. The corresponding decomposition of

3) In [7], we stated that the decomposition of $T_{p}$ of this kind is unique without the assumption that $M$ is simply connected. But this is not exact. In Theorem 2, therefore, we have added the assumption of simply-connectedness which was not made in [7]. 
$M$ which is assured by $A$ is called the canonical decomposition of the Riemannian manifold $M$. $T_{0}$ ( or $M_{0}$ ) is called the Euclidean part of $T_{p}$ (or $M$ ).

We now prove the following main lemma.

Lemma 5. Let $M$ be simply connected and complete, and let $T=T_{0}+T_{1}$ $+\ldots+T_{r}$ be the canonical decomposition of $T_{p}$. If $\varphi \in A^{0}(V)$, then $s(\varphi) \cdot$ $T_{i}=T_{i}$ for every $i=0,1, \ldots, r$.

Proof. Since $A^{0}(V)$ is a connected Lie group, it is sufficient, by virtue of Lemma 3 , to prove Lemma 5 for $\varphi$ sufficiently near to the identity element. We may assume $\varphi$ to lie on a one-parameter group $\varphi_{t}$. By using $\rho_{t}$ in Lemma 4, we shall show that $\rho_{t} \cdot T_{i}=T_{i}$ for every $i$. First of all, $\Psi_{p}$ acts trivially on $\rho_{t} \cdot T_{0}$ as is clear from the equivalence of $T_{0}$ and $\rho_{t} \cdot T_{0}$ which we explained before. Therefore, $\rho_{t} \cdot T_{0} \subset T_{0}$ and, indeed, $\rho_{t} \cdot T_{0}=T_{0}$. For each $i=1,2, \ldots, r, \rho_{t} \cdot T_{i}$ is an invariant subspace and, by the result $B$ of de Rham, it is either orthogonal to $T_{j}$ or contains $T_{j}(j=1,2, \ldots, r)$. If $\rho_{t} \cdot T_{i}$ is orthogonal to every $T_{j}$, $j=1,2, \ldots, r$, then $\rho_{t} \cdot T_{i}$ must be contained in $T_{0}$, which is a contradiction. Therefore, $\rho_{t} \cdot T_{i}$ contains some $T_{j}(j=1,2, \ldots, r)$. Again by the equivalence of $T_{i}$ and $\rho_{t} \cdot T_{i}, \rho_{t} T_{i}$ is irreducible and hence coincides with $T_{j}$.

By this argument, we have seen that each $\rho_{t}$ maps $T_{0}$ into itself and $T_{i}$ into some $T_{j}(i, j=1,2, \ldots, r)$. We now show that $\rho_{t} \cdot T_{i}=T_{i}$ for sufficiently small $t$; then this is true for every $t$. Let $X$ be any element $\neq 0$ of $T_{i} . \rho_{t} \cdot X$ must belong to some $T_{j}$ as we have already shown. Since $\left(\rho_{t} X, X\right)$ is continuous in $t$ and is not 0 for $t=0$, it is not zero for sufficiently small $t$. For such $t, \rho_{t} \cdot X$ cannot belong to $T_{j}(j \neq i)$ which is orthogonal to $T_{i}$. This means that there is $\delta>0$ such that if $|t|<\delta$, then $\rho_{t} \cdot X \in T_{i}$. Now by taking a base of $T_{i}$, we see that there is $\delta^{\prime}>0$ such that if $|t|<\delta^{\prime}$ then $\rho_{t}$ maps every element of the base into $T_{i}$. By linearity, $\rho_{t}$ maps $T_{i}$ into $T_{i}$. This concludes the proof of Lemma 5 .

3. Affine transformations and isometries. If $M$ is irreducible, then there is a homomorphism $c(\varphi)$ of $A(M)$ into the multiplicative group of positive numbers such that $|\varphi X|=c(\varphi)|X|$, for every tangent vector $X$, as we have seen in Lemma 2. If $c(\varphi)=1$, then $\varphi$ is an isometry. We have therefore

Theorem 1. Let $M$ be an irreducible Riemannian manifold. Then the following subgroups of $A(M)$ are all contained in the group of isometries $I(M)$ : 
1) every compact subgroup of $A(M)$;

2) every connected semi-simple Lie subgroup of $A(M)$;

3) the commutator subgroup $[A(M), A(M)]$.

COROLlaRy. If $M$ is a compact irreducible Riemannian manifold, then $I(M)$ is the largest compact subgroup of $A(M){ }^{4)}$

In order to deal with reducible $M$, we assume $M$ to be simply connected and complete. We take the canonical decomposition $T_{p}=T_{0}+T_{1}+\ldots+T_{r}$ and the corresponding distributions $\left(T_{i}\right)$ obtained from $T_{i}$ by parallel displacement. Lemma 5 implies that $\varphi \cdot T_{i}=\left(T_{i}\right)_{\varphi(p)}$ for every $\varphi \in A^{0}(M)$. We now show that, for each $i=1, \ldots, r$, there is a homomorphism $\varphi \rightarrow c_{i}(\varphi)$ of $A^{0}(M)$ into the multiplicative group of positive numbers such that $|\varphi X|=c_{i}(\varphi)|X|$ for any tangent vector $X$ belonging to the distribution $\left(T_{i}\right)$. Let us fix $i$. Since $\Psi_{p}$ is irreducible on $T_{i}$, we see by the same argument as in Lemma 2 that there is a positive constant $c_{i}$ such that $|\varphi X|_{p(p)}=c_{i}|X|_{p}$ for every $X \in T_{i}$. Now if $Y$ is a tangent vector belonging to $\left(T_{i}\right)_{q}$, then we take a curve from $q$ to $p$ and we have $|\varphi \cdot Y|=|\varphi(\tau) \cdot \varphi Y|=|\varphi \cdot \tau Y|=c_{i}(\varphi)|\tau \cdot Y|=c_{i}(\varphi)|Y|$, because $\tau Y$ $\in T_{i}$. We show that $\varphi \in A^{0}(M) \rightarrow c_{i}(\varphi)$ is a homomorphism. If $\varphi, \psi \in A_{0}(M)$ and if $X$ is a tangent vector belonging to the distribution $\left(T_{i}\right)$, then $\psi X$ belongs to $\left(T_{i}\right)$ and we have $|\varphi \cdot \psi X|=c_{i}(\varphi)|\psi X|=c_{i}(\varphi) c_{i}(\psi)|X|$.

We have thereby proved

THEOREM 2. If $M$ is a simply connected complete Riemannian manifold whose Euclidean part is of dimension $\leqq 1$, then the following subgroups of $A^{0}(M)$ are all contained in $I(M)$ :

1) every compact subgroup of $A^{0}(M)$;

2) every connected semi-simple Lie subgroup of $A^{0}(M)$;

3 ) the commutator subgroup $\left[A^{0}(M), A^{0}(M)\right]$.

\section{Riemannian homogeneous spaces}

4. Riemannian homogeneous space. Universal covering. A Riemannian manifold $M$ is called Riemannian homogeneous if the largest connected group of isometries $I^{0}(M)$ is transitive on $M$. In this case, we can represent $M$ as a homogeneous space $G / H$ with a $G$-invariant Riemannian metric on it, where $G$

4) It might be remarked that $A(M)$ and $I(M)$ have the same identity component by a result of Yano r12]. 
is a connected Lie group and the isotropy subgroup $H$ is compact. A Riemannian homogeneous space is always complete.

The universal covering manifold of $M=G / H$ is Riemannian homogeneous with respect to a natural Riemannian metric. Let $\widetilde{G}$ be the universal covering group of $G$ and let $\widetilde{H}$ be the identity component of the complete inverse image of $H$ by the canonical projection $\pi$ of $\widetilde{G}$ onto $G$. $\widetilde{H}$ is closed and $\widetilde{G} / \widetilde{H}$ is nothing but the universal covering manifold $\tilde{M}$ of $M$. $\pi$ induces the canonical projection of $\widetilde{M}$ onto $M$, denoted by the same letter, such that $\pi(\widetilde{a} \cdot \tilde{p})=\pi(\widetilde{a}) \cdot \pi(\tilde{p})$ for $\widetilde{a} \in \widetilde{G}$ and $\widetilde{p} \in \tilde{M}$. Now $\pi$ being a covering mapping, we can transfer the Riemannian metric on $M$ to $\tilde{M}$ in such a way that $\pi$ is a local isometry, that is, every $\widetilde{p} \in \tilde{M}$ has a neighborhood on which $\pi$ is an isometry. Then it is easy to see that every $\tilde{a} \in \widetilde{G}$ is an isometry of $\tilde{M}$. This proves that $\widetilde{M}=\widetilde{G} / \widetilde{H}$ is Riemannian homogeneous.

In general, the universal covering manifold $\tilde{M}$ of a Riemannian manifold $M$ has a (unique) Riemannian metric such that the canonical projection $\pi$ of $\tilde{M}$ onto $M$ is a local isometry. If $M$ is complete, so is $\tilde{M}$. The restricted homogeneous holonomy group $\Psi^{0}(M)$ of $M$ is isomorphic with the homogeneous holonomy group $\Psi(\tilde{M})$ of $\tilde{M}$ [1]. More precisely, if $\tilde{\tau}$ is an element of $\Psi(\tilde{M})$ represented by a closed curve at $\widetilde{p}$ of $\tilde{M}$, then $\pi \cdot \widetilde{\tau}=\tau \cdot \pi$, where $\tau$ is the element of $\Psi^{0}(M)$ represented by the closed curve $\tau=\pi(\widetilde{\tau})$ at $p=\pi(\widetilde{p})$ of $M$. Conversely, for every $\tau \in \Psi^{0}(M)$ there is $\tilde{\tau} \in \Psi(\tilde{M})$ satisfying the above relation and this correspondence is one-to-one.

Lemma 6. Let $\tilde{p} \in \tilde{M}$ and $\pi(\tilde{p})=p \in M$. For any $\varphi \in I(M)$ such that $\varphi(p)$ $=p$, there is a $\tilde{\varphi} \in I(\tilde{M})$ such that $\tilde{\varphi}(\tilde{p})=\tilde{p}$ and $\pi \cdot \tilde{\varphi}=\varphi \cdot \pi$.

Proof. We may consider $\tilde{M}$ as the set of homotopy classes of curves of $M$ issuing from $p$ and assume $\widetilde{p}$ to be the homotopy class of the trivial curve consisting of $p$ only. If $\tilde{q}$ is any point of $\tilde{M}$ represented by a curve $\tau$ from $p$ to $q=\pi(\tilde{q})$, then we define $\tilde{\varphi} \cdot \tilde{q}$ to be the homotopy class of the curve $\varphi(\tau)$ from $p$ to $\varphi(q)$; this definition is legitimate, because the homotopy class of $\varphi(\tau)$ is determined by that of $\tau$. We have clearly $\pi \cdot \widetilde{\varphi}=\varphi \cdot \pi$ and $\tilde{\varphi}(\tilde{p})=\tilde{p}$. It is easy to verify that $\tilde{\varphi}$ is an isometry of $\tilde{M}$.

Remark. If $\varphi_{t}$ is a 1-parameter group of isometries of $M$, then there is a 1-parameter group of isometries $\widetilde{\varphi}_{t}$ of $\tilde{M}$ such that $\pi \cdot \widetilde{\varphi}_{t}=\varphi_{t} \cdot \pi$. (This may be 
proved by a similar method, or by considering the killing vector field induced by $\varphi_{t}$.)

5. Canonical decomposition of $\boldsymbol{G} / \boldsymbol{H}$. We shall prove

Theorem 3. Let $G / H$ be a simply connected Riemannian homogeneous space. Then each factor of the canonical decomposition of $G / H$ is Riemannian homogeneous. More precisely, there exist connected closed subgroups $G_{0}, G_{1}, \ldots, G_{r}$ of $G$, all containing $H$, such that $G_{0} / H \times G_{1} / H \times \ldots \times G_{r} / H$ is the canonical decomposition of $\mathrm{G} / \mathrm{H}$.

Proof. Let $p$ be the point of $G / H$ represented by the coset $H$, and let $T_{p}$ $=T_{0}+T_{1}+\ldots+T_{r}$ be the canonical decomposition of the tangent space. $G$ being connected, we see from Lemma 5 that $a \cdot T_{1}=\left(T_{i}\right)_{a \cdot p}$ for every $a \in G$ and $i=0,1, \ldots, r$. It follows that each distribution $\left(T_{i}\right)$ is invariant by $G$. Let $M_{i}$ be the maximal integral manifold of $\left(T_{i}\right)$ through $p$. We show that $M_{i}$ is Riemannian homogereous. Any point of $M_{i}$ may be written as $a \cdot p$ for some $a \in G$. Then $a \cdot M_{i}$ is the maximal integral manifold of $\left(T_{i}\right)$ through $a \cdot p$ and hence coincides with $M_{i}$. This means that $a \cdot M_{i}=M_{i}$. Therefore a is an isometry of $M_{i}$ which maps $p$ into the point $a \cdot p$.

Let $G_{i}$ be the set of $a \in G$ such that $a \cdot p \in M_{i}$. Since it is the set of $a \in G$ such that $a \cdot M_{i}=M_{i}$, it is a closed subgroup of $G$ (remark that each $M_{i}$ is closed in $G / H$ ). We show that $G_{i}$ is connected. Let a be any element of $G_{i}$, and take a curve $p(t)$ in $G / H$ such that $p(0)=p$ and $p(1)=a \cdot p$. The curve $p(t)$ may be written as $p(t)=a(t) \cdot p$ where $a(t)$ is a curve in $G$ such that $a(1)=a$ and $a(0)$ is a certain element of $H$. Since $a_{t} \cdot p$ belongs to $M_{i}, a_{t}$ belongs to $G_{i}$. Since $G / H$ is simply connected, $H$ is connected. It follows that $a(0)$ and hence $a(1)$ may be joined to the identity element by a curve in $G_{i}$, which proves that $G_{i}$ is connected. This concludes the proof of Theorem 3 .

TheOREM 4. Let $\mathrm{G} / \mathrm{H}$ be a simply connected Riemannian homogeneous space. If the linear isotropy group $\widetilde{H}$ is irreducible, then either $G / H$ is euclidean or $G / H$ is irreducible and non-euclidean.

Proof. By Lemma 5 and by the remark at the end of 1, I, we know that each factor of the canonical decomposition of the tangent space $T_{p}=T_{0}+T_{1}$ $+\ldots+T_{r}$ is invariant by $\tilde{H}$. 
Corollary. Let $G / H$ be a Riemannian homogeneous space. If the connected linear isotropy group $\widetilde{H}^{0}$ is irreducible, then either $G / H$ is locally euclidean or the restricted homogeneous holonomy group is irreducible and non-trivial.

Proof. We take the universal covering of $G / H$ and apply Theorem $4 .^{5)}$

6. A theorem on forms whose covariant derivatives are zero. In any compact Riemannian manifold $M$, a harmonic form $\omega$ is invariant by $I^{0}(M)$ [12]. This may be proved topologically as follows. It is sufficient to prove the invariance of $\omega$ by any one-parameter subgroup of isometries $\varphi_{t}$. It is clear that the transform $\varphi_{t}^{*} \cdot \omega$ is again harmonic. For any cycle $C$ of the same dimension as $\omega$, the integral $\int_{C} \varphi_{t}{ }^{*} \cdot \omega$ is equal to $\int_{\varphi_{t \cdot c}} \omega$, where $\varphi_{t} \cdot C$ is the image of $C$ by $\varphi_{t}$ and is homologous to $C$. Therefore $\int_{C} \varphi_{t}^{*} \omega=\int_{C} \omega$ for every cycle $C$, which implies that $\varphi_{t}^{*} \cdot \omega=\omega$ by a fundamental theorem of Hodge.

We shall prove

Theorem 5. ${ }^{6)}$ Let $G / H$ be a Riemannian homogeneous space. If $G$ is simple, any form $\omega$ on $G / H$ such that $\nabla \omega=0$ is invariant by $G$.

Proof. Let $p$ be the point represented by the coset $H$. Let $A$ be the exterior algebra over the dual of the tangent space $T_{p}$. The Euclidean metric on $T_{p}$ may be extended to a Euclidean metric on $A$, and the homogeneous holonomy group $\Psi_{p}$ may be extended to an orthogonal group of $A$ in the natural fashion. Let $A^{\prime}$ be the subspace of $A$ consisting of all elements of $A$ which are invariant by $\Psi_{p}$. There is a one-to-one correspondence between $A^{\prime}$ and the set of differential forms $\omega$ on $G / H$ with $\nabla \omega=0$. Such an $\omega$ is obtained from some element of $A^{\prime}$ by the parallel displacement.

This being said, let a be any element of $G$ and let $\tau$ be any curve from $p$ to $a \cdot p$. We consider the linear transformation $\tau^{-1} \cdot a$ of $T_{p}$ as we did in 1 , I. By a similar argument, we can easily show that the induced linear transformation $\tau^{-1} \cdot a$ of $A$ leaves $A^{\prime}$ invariant and that the induced linear transformation of $A^{\prime}$ does not depend on the choice of $\tau$. We shall denote by $u(a)$ the linear transformation of $A^{\prime}$ which is so defined. Since $a$ is an isometry of

5) This poof was suggested by J. Hano.

6) The assumption that the center of $G$ consists of the identity only [8] has been removed by a suggestion of J. Hano. 
$G / H, \tau^{-1} \cdot a$ is an isometry of $T_{p}$. Hence $u(a)$ is an isometry of $A^{\prime}$. Finally, it is easy to prove that $a \rightarrow u(a)$ is a homomorphism.

In this way, we have a representation of $G$ into the orthogonal group of $A^{\prime}$. It is not difficult to show that this representation is continuous. Since $G$ is simple, the kernel $K$ of this representation coincides with $G$ or is a discrete invariant subgroup. In the first case, $u(a)$ is the identity on $A^{\prime}$ for every $a \in G$. This means that $a \cdot f=\tau \cdot f$ for every $f \in A^{\prime}$, where $\tau$ is the parallel displacement along any curve from $p$ to $a \cdot p$. If $\omega$ is a differential form obtained by the parallel displacement of $f$, then $\omega$ is invariant by $a$. In the second case, $G / K$ admits a faithful representation into the orthogonal group of $A^{\prime}$. It follows that $G / K$ is maximally almost periodic and hence compact. ${ }^{7)}$ This is the same for $G$. If $G / H$ is compact, Theorem 5 is a trivial consequence of the proposition on harmonic forms.

\section{Holonomy algebra of $G / H$}

7. The general case. Let $G / H$ be a reductive homogeneous space with a decomposition of the Lie algebra $\mathfrak{g}=\mathfrak{m}+\mathfrak{h}, a d(H) \mathfrak{m}=\mathfrak{m}$. We have shown previously [6] that there is a one-to-one correspondence between the set of invariant affine connections on $G / H$ and the set of bilinear functions $\alpha(X, Y)$ on $\mathfrak{m} \times \mathfrak{m}$ with values in $m$ which are compatible with $a d(H): a d(h) \cdot \alpha(X, Y)=\alpha(a d(h) X$, $\boldsymbol{a d}(\boldsymbol{h}) Y)$. We shall determine the holonomy algebra, that is, the Lie algebra of the restricted homogeneous holonomy group of an invariant affine connection.

We first recall the formulas for the curvature tensor and its successive covariant derivatives [6]. For each $X \in \mathfrak{m}$ we define a linear endomorphism $\rho(X)$ of $\mathrm{m}$ by $\rho(X) \cdot \mathrm{Y}=\alpha(X, Y)$. Then the curvature tensor is given by

$$
R(X, Y)=[\rho(X), \rho(Y)]-\rho\left([X, Y]_{\mathfrak{m}}\right)-a d\left([X, Y]_{\mathfrak{h}}\right),
$$

where $[X, Y]_{\mathfrak{m}}$ (resp. $\left.[X, Y]_{\mathfrak{y}}\right)$ denotes the $\mathfrak{n}$-component (resp. $\mathfrak{h}$-component) of $[X, Y] \in \mathfrak{g}$. The covariant derivative of $R$ is given by

$$
(\nabla R)(X, Y: Z)=\left(\nabla_{Z} R\right)(X, Y)=[\rho(Z), R(X, Y)]-R(\rho(Z) X, Y)-R(X, \rho(Z) Y),
$$

and similarly for the successive covariant derivatives.

7) Any maximally almost periodic Lie group is the direct product of a compact group and an abelian group isomorphic with the additive group $R^{n}$. Here $G$ is simple and hence compact. 
Since $G / H$ is homogeneous, the holonomy algebra, denoted by $\mathfrak{h}^{*}$, is generated by the endomorphisms of $m$ of the form $R(X, Y),(\nabla R)(X, Y ; Z)$, $\left(\nabla^{2} R\right)(X, Y ; Z ; W), \ldots$ (all successive covariant derivatives), where $X, Y, Z$, $W, \ldots$ run through $m$ (Theorem $7,[5])^{8)}$

Lemma 7 (Chevalley). The holonomy algebra is equal to the smallest Lie algebra of endomorphisms of $\mathfrak{m}$ such that 1) $R(X, Y) \in \mathfrak{h}^{*}$ for all $X, Y \in \mathfrak{m}$ and 2) $\left[\rho(X), \mathfrak{h}^{*}\right] \subset \mathfrak{h}^{*}$ for all $X \in \mathfrak{m}$.

Proof. Let $\mathfrak{h}^{* *}$ be the smallest algebra satisfying 1) and 2). First we show that the holonomy algebra $\mathfrak{h}^{*}$ satisfies 1 ) and 2$)$; then $\mathfrak{h}^{*} \supset \mathfrak{h}^{* *}$. The condition 1 ) is obvious. In order to prove 2$)$, it is sufficient to prove that $[\rho(X), A] \in \mathfrak{h}^{*}$ for every generator $A$ (one of the successive covariant derivatives of $R$ ) of $\mathfrak{h}^{*}$ and for every $X \in \mathfrak{m}$. In fact, the set of elements $S$ of $\mathfrak{h}^{*}$ such that $[\rho(X), S] \in \mathfrak{h}^{*}$ for every $X \in \mathfrak{m}$ forms a subalgebra $\mathfrak{H}^{*}$ of $\mathfrak{h}^{*}$. If every generator $A$ of $\mathfrak{h}^{*}$ belongs to $\mathfrak{H}^{*}$, then $\mathfrak{h}^{*}$ is contained in $\mathfrak{k}$, that is, $\left[\rho(X), \mathfrak{h}^{*}\right] \subset \mathfrak{h}^{*}$. Now if $A=R(Y, Z)$, then

$$
[\rho(X), R(Y, Z)]=\left(\nabla_{X} R\right)(Y, Z)+R(\rho(X) \cdot Y, Z)+R(Y, \rho(X) \cdot Z)
$$

belongs to $\mathfrak{h}^{*}$. The similar reasoning applies to any other generator $A$. We have thus proved that $\mathfrak{h}^{* *} \subset \mathfrak{h}^{*}$.

In order to show that $\mathfrak{h}^{* *} \supset \mathfrak{h}^{*}$, it is sufficient to verify that $R(X, Y)$, $(\nabla R)(X, Y ; Z), \ldots$, belong to $\mathfrak{h}^{* *}$. $R(X, Y)$ belong to $\mathfrak{h}^{* *}$ by the condition 1$)$. As for $(\nabla R)(X, Y ; Z)$, we have

$$
(\nabla R)(X, Y ; Z)=[\rho(Z), R(X, Y)]-R(\rho(Z) X, Y)-R(X, \rho(Z) Y),
$$

where $[\rho(Z), R(X, Y)] \in \mathfrak{h}^{* *}$ by the condition 2) and $R(\rho(Z) X, Y), R(X, \rho(Z) Y)$ $\in \mathfrak{h}^{* *}$ by 1$)$. Hence $(\nabla R)(X, Y ; Z) \in \mathfrak{h}^{* *}$. Similarly, all the successive covariant derivatives of $R$ belong to $\mathfrak{h}^{* *}$. This concludes the proof of Lemma 7 .

Let $\mathfrak{h}_{1}$ be the linear subspace of $\mathfrak{h}$ spanned by all elements of the form $[X, Y]_{\mathfrak{h}}$, where $X, Y \in \mathfrak{m}$. $\mathfrak{h}_{1}$ is an ideal of $\mathfrak{h}$.

LEMMA 8. If the normalisor of $\mathfrak{h}^{*}$ coincides with $\mathfrak{h}^{*}$, then $\mathfrak{h}^{*}$ is generated by $\rho(X), X \in \mathfrak{m}$, and $\operatorname{ad}\left(\mathfrak{h}_{1}\right)$.

Proof. In this case, the condition 2) implies that $\rho(X) \in \mathfrak{h}^{*}$ for every $X$ $\in \mathfrak{m}$. We denote by $\mathfrak{h}^{* *}$ the subalgebra generated by $\operatorname{ad}\left(\mathfrak{h}_{1}\right)$ and $\rho(X), X \in \mathfrak{m}$.

\footnotetext{
8) It is easy to prove that any invariant affine connction on $G / H$ is analytic.
} 
Then $R(X, Y)$ belongs to $\mathfrak{h}^{* *}$ as is clear from the formula. Of course, $\left[\rho(X), \mathfrak{h}^{* *}\right]$ $\subset h^{* *}$ for every $X \in \mathrm{m}$. Hence $\mathfrak{h}^{* *}$ satisfies the conditions 1) and 2) of Lemma 7 and hence $\mathfrak{h}^{* *} \supset \mathfrak{h}^{*}$. On the other hand, we see that $\mathfrak{h}^{*}$ contains $\operatorname{ad}\left([X, Y]_{\mathfrak{n}}\right)$ for all $X, Y \in m$, and hence $a d\left(\mathfrak{h}_{1}\right)$. Therefore $\mathfrak{h}^{* *} \subset \mathfrak{h}^{*}$. We have proved tha: $\mathfrak{h}^{* *}=\mathfrak{h}^{*}$.

8. The irreducible case. Let $G / H$ be a homogeneous space of a connected Lie group $G$ with compact $H$. If the connected linear isotropy group $\widetilde{H}^{0}$ is irreducible, then there is an invariant Riemannian metric on $G / H$ which is uniquely determined up to a constant factor. The associated Riemannian connection is then unique. We shall determine the holonomy algebra of $G / H$ in the essential cases.

If $G / H$ is not locally Euclidean, we see from the corollary to Theorem 4 that the restricted homogeneous holonomy group is irreducible. By a result of Borel-Lichnerowicz [3], the normalizor of the holonomy algebra $\mathfrak{h}^{*}$ coincides with $\mathfrak{h}^{*}$ if $G / H$ is not Kähleran. If $G / H$ is a Kählerian space whose Ricci curvature tensor is not zero, then it is hermitian symmetric, again by a result of Lichnerowicz [2].

From these considerations and from Lemma 8, we have the algebraic determination of the holonomy algebra of $G / H$ except the case where $G / H$ is Kählerian with zero Ricci curvature.

Thorem 6. Let $\mathrm{G} / \mathrm{H}$ be a Riemannian homogeneous space which is not locally Euclidean and whose connected linear isotropy group is irreducible. If $G / H$ is not Kählerian, the holonomy algebra is generated by $\rho(X), X \in \mathfrak{m}$, and ad $\left(\mathfrak{h}_{1}\right)$. If it is Kählerian and if the Ricci curvature tensor is not zero, it is hermitian symmetric and the holonomy algebra is isomorphic with ad $\left(\mathfrak{h}_{1}\right)$.

In the case where $G$ is compact, there is a suitable decomposition of the Lie algebra $\mathfrak{g}=\mathrm{m}+\mathfrak{h}, a d(H) \mathfrak{m}=\mathrm{m}$, with respect to which the invariant Riemannian connection is given by $\alpha(X \cdot Y)=(1 / 2)[X, Y]_{\mathfrak{m}}$. Indeed, we can take a positive definite quadratic form on $g$ which is invariant by $\operatorname{ad}(G)$ and take the orthogonal complement $m$ to $\mathfrak{h}$. Then the induced metric on $m$ gives rise to an invariant Riemannian metric on $G / \widetilde{H}$, which induces the unique invariant Riemannian connection on $G / H$ since $\widetilde{H}$ is irreducible. For this invariant Riemannian metric, the corresponding connection is given by $\alpha(X, Y)=(1 / 2)[X, Y]_{\mathfrak{m}}$ (Theorem 13.2., [6]). 
Furthermore, if the Ricci curvature is zero, then $G / H$ is locally Euclidean [3]. We have hence

Theorem 7. Let $G / H$ be a Riemannian homogeneous space with compact $G$ and with irreducible $\widetilde{H}^{0}$. If $G / H$ is not locally Euclidean, the holonomy algebra is generated by $\rho(X), X \in \mathrm{m}$, and ad $\left(\mathfrak{h}_{1}\right)$, where, in a suitable decomposition of the Lie algebra $\mathfrak{g}=\mathrm{m}+\mathfrak{h}$, ad $(H) \mathrm{m}=\mathrm{m}, \rho(X)$ is the linear transformation of $\mathrm{m}$ defined by $\rho(X) \cdot Y=(1 / 2)[X, Y]_{\mathfrak{m}}$ and $\mathfrak{h}_{1}$ is the subspace (indeed, an ideal) of $\mathfrak{h}$ spanned by all elements of the form $[X, Y]_{\mathfrak{n}}$.

\section{BIBLIOGRAPHY}

[1] A. Borel and A. Lichnerowicz, Groupes d'holonomie des varietés riemanniennes, Comptes rendus, Paris, 234 (1952), 1835-1837.

[2] A. Lichnerowicz, Variétés pseudokählériennes à courbure de Ricci non nulle; application aux domaines bornés homogènes de $C^{n}$, Comptes rendus, Pairs, 234 (1952), 12-14.

[3] A. Lichnerowicz, Espaces homogènes kählériens, Colloque de Géométrie Différentielle, Strasbourg, (1953), 171-184.

[4] A. Morimoto and J. Hano, Note on the group of affine transformations of an affinely connected manifold, Nagoya Math. Jour. 8 (1955), 85-95.

[5] A. Nijenhuis, On the holonomy groups of linear connections, Koninkl. Nederl. Akademie van Wetenschappen, Amsterdam, Proc., Series A, 56 (1953), 233-249.

[6] K. Nomizu, Invariant affine connections on homogeneous spaces, Amer. Journ. Math., 76 (1954), 33-65.

[7] K. Nomizu, Sur les transformations affines d'une variété riemannienne, Comptes rendus, Paris, 237 (1953), 1308-1310.

[8] K. Nomizu, Application de l'étude des transformations affines aux espaces homogènes riemanniens, Comptes rendus, Paris, 237 (1953), 1386-1387.

[9] K. Nomizu, Sur l'algèbre d'holonomie d'un espace homogène riemannien, Comptes rendus, Paris, 238 (1954), 319-321.

[10] K. Nomizu, Remarques sur les groupes d'holonomie et disotropie, Colloque de Topologie de Strasbourg (mai, 1954).

[11] G. de Rham, Sur la réductibilité d'un espace de Riemann, Comment. Math. Helv., 26 (1952), 328-344.

[12] K. Yano, On harmonic and Killing vector fields, Ann. of Math. 55 (1952), 38-45.

\section{Mathematical Institute, \\ Nagoya University}

Added in proof. For the group of affine transformations, see the papers of J. Hano and S. Kobayashi (in this journal) which give more complete results than $\mathrm{I}$ of this paper. 